\title{
ПОДХОД К АНАЛИЗУ ВОЗМОЖНОЙ СТЕПЕНИ СБАЛАНСИРОВАННОСТИ СПРОСА И ПРЕДЛОЖЕНИЯ ТРУДА ИНЖЕНЕРНО-ТЕХНИЧЕСКИХ СПЕЦИАЛИСТОВ
}

\author{
Кочеткова Е.В.
}

В работе предложена макроэкономическая модель для исследования и прогнозирования спроса и предложения труда инженерно-технических специалистов. Показана возможность использования данной модели для проведения сиенарного прогноза численности занятых инженернотехнических специалистов и получения оценки возможной степени сбалансированности рынка труда инженерно-технических специалистов на период 2020-2024 г2.

DOI: $10.20537 /$ mce2020econ16

Введение. В настоящее время для многих стран характерна ситуация несбалансированности спроса и предложения труда инженернотехнических специалистов (ИТС). Это явление может принимать различные формы: дефицит кадров, в том числе нехватки опытных инженерных работников конкретных профессий, трудности в поиске работы для молодых специалистов, претензии работодателей к качеству профессионального образования и т.п. (см., например, [1]).

По причине отсутствия общепризнанного однозначного статистического показателя дефицита или избытка рабочей силы [2], в данной работе предлагается под сбалансированностью спроса и предложения понимать состояние рынка труда ИТС, при котором спрос на труд ИТС может быть обеспечен за счет внутреннего предложения труда ИТС, главным источником которого является выпуск специалистов.

При анализе степени сбалансированности спрос на труд ИТС $\left(D E T_{t}\right)$ был определен как дополнительная потребность в ИТС (аналогичный подход предложен в [3]) в соответствии со следующим уравнением:

$$
D E T_{t}=E T_{t}-(1-m) E T_{t-1}
$$

где $E T_{t}$ - численность занятых ИТС, $m=0.033$ - коэффициент, характеризующий дополнительную потребность, связанную с выбытием кадров и появлением вакантных мест, величина которого определена на ос- 
нове данных Росстата [4] за 2008-2018 гг. с учетом результатов, полученных в [5] (введен аналогично коэффициентам естественной ротации и выбытия из-за смены вида деятельности, см. [6], но без конкретизации отдельных видов выбытия).

Предложение труда ИТС $\left(S G E_{t}\right)$ при анализе сбалансированности оценивалось как выпуск специалистов со средним и высшим инженернотехническим образованием (аналогичным образом предлагается рассматривать предложение квалифицированных кадров в работе [7]) с учетом доли специалистов, не работающих по полученной специальности:

$$
S G E_{t}=(1-\zeta) G E_{t},
$$

где $\xi$ - доля специалистов, не работающих по специальности после окончания вуза/ссуза, $G E_{t}$ - численность выпуска ИТС из вузов и ссузов.

Для оценки степени сбалансированности спроса и предложения ИТС возможно использовать соотношение полученных оценок объема дополнительного спроса на ИТС $\left(D E T_{t}\right)$ и предложения труда ИТС $\left(S G E_{t}\right)$ : $\left(S G E_{t} / D E T_{t}\right)$. Величина данного отношения менее 1 свидетельствует о возможном дефиците подготавливаемых кадров, выше 1 - о возможной безработице и потере кадрового потенциала ИТС.

Модель. Для моделирования динамики и анализа степени сбалансированности спроса и предложения ИТС была разработана макроэкономическая модель, состоящая из 12 блоков.

Выходными переменными в модели являются численность выпуска ИТС $\left(G E_{t}\right)$, численность занятых ИТС $\left(E T_{t}\right)$, экзогенными - численность занятых в экономике $\left(L_{t}\right)$; численность выпуска специалистов со средним и высшим профессиональным образованием по всем специальностям и направлениям подготовки $\left(G_{t}\right)$, уровень загрузки производственныХ мощностей $\left(Z_{t}\right)$, доля инвестиций в ВВП $\left(a_{t}\right)$, доля инвестиций в основные фонды (ОФ) промышленности $\left(a i_{t}\right)$ и доля инвестиций в ОФ сектора услуг $\left(a s_{t}\right)$ в инвестициях в целом.

Блоки макроэкономической модели заданы уравнениями:

1. численность выпуска ИТС

$$
\begin{aligned}
& \Delta g e_{t} / g e_{t-1}=c_{1}+c_{2} g e_{t-1}+c_{3} \Delta g e_{t-1}+c_{4} g s_{t-1}+c_{5} \Delta Y i_{t-5} / Y i_{t-6}+ \\
& +c_{6} D 1+c_{7} D 2 \\
& \Delta g s_{t} / g s_{t-1}=c_{8}+c_{9} g e_{t-1}+c_{10} g s_{t-1}+c_{11} \Delta Y s_{t-6} / Y s_{t-7} \\
& G E_{t}=g e_{t} G_{t}
\end{aligned}
$$


где $g e_{t}$ - доля выпускников инженерно-технических специальностей в общем выпуске, \%; $g s_{t}$ - доля выпускников социально-гуманитарных специальностей в общем выпуске, \%; $G E_{t}$ - численность выпуска ИТС, тыс. человек; $Y i_{t}$ - объем выпуска промышленности, млрд руб., $Y_{s_{t}}$ - валовая добавленная стоимость в секторе услуг, млрд руб.; $G_{t}-$ численность выпуска специалистов со средним и высшим профессиональным образованием по всем специальностям и направлениям подготовки, тыс. человек; фиктивные переменные D1 = 1 в 2013-2014 гг. и 0 в остальных случаях, D2 = 1 в 2018 г. и 0 в остальных случаях;

2. численность занятых ИТС

$$
\begin{aligned}
& e t_{t}=c_{12} g e_{t}+c_{13} w_{t}+c_{14} D 3, \\
& E T_{t}=e t_{t} L_{t},
\end{aligned}
$$

где $e t_{t}$ - доля численности занятых ИТС в общей численности занятых в экономике, \%, $E T_{t}$ - численность занятых ИТС в экономике, тыс. человек, $L_{t}$ - численность занятых в экономике, тыс. человек, $w_{t}-$ отношение средней заработной платы в промышленности к средней заработной плате в финансовой деятельности, \%; фиктивная переменная D3 = 1 в 2016 г. и 0 в остальных случаях;

3. производственная функция промышленности

$$
Y i_{t}=c_{15}\left(F i_{t} Z_{t}\right)^{c_{16}}\left(L i_{t}\right)^{1-c_{16}},
$$

где $Z_{t}$ - уровень загрузки производственных мощностей в промышленности, \%; $F i_{t}$ — основные фонды в промышленности, млрд руб.; $L i_{t}-$ численность занятых в промышленности, тыс. человек;

4. производственная функция экономики в целом

$$
\begin{aligned}
& Y_{t}=c_{17} F_{t}^{c_{18}} L_{t}^{1-c_{18}}, \\
& d \ln T F P_{t}=c_{19}+c_{20} d \ln \left(Y i_{t} / L i_{t}\right),
\end{aligned}
$$

где $Y_{t}-$ ВВП, млрд руб.; $F_{t}$ - основные фонды экономики, млрд руб.; $T F P_{t}$ - совокупная производительность факторов производства (total factor productivity).

5. производственная функция сектора услуг

$$
Y s_{t}=c_{21} F s_{t}^{c_{22}} L s_{t}^{1-c_{22}} C_{t}^{c_{23}} \text {, }
$$

где $F s_{t}$ - основные фонды в секторе услуг, млрд руб.; $L s_{t}$ - численность занятых в секторе услуг, тыс. человек; $C_{t}$ - совокупные расходы на потребление, млрд руб.; 
6. численность занятых в промышленности

$$
\begin{aligned}
& l i_{t}=c_{24} l i_{t-1}+c_{25}\left(F i_{t-1} Z_{t-1}\right), \\
& L i_{t}=l i_{t} L_{t},
\end{aligned}
$$

где $l i_{t}$ - доля занятых в промышленности, \%;

7. численность занятых в секторе услуг

$$
\begin{aligned}
& l s_{t}=c_{26}\left(Y_{s_{t-1}} / Y_{t-1}\right)+c_{27} l i_{t}+c_{28} D 4, \\
& L s_{t}=l s_{t} L_{t},
\end{aligned}
$$

где $l s_{t}$ - доля занятых в промышленности, \%; фиктивная переменная D4 = 1 в 2015 и 2016 гг. и 0 в остальных случаях;

8. основные фонды (ОФ) в экономике

$$
\begin{aligned}
& F_{t}=c_{29} F_{t-1}+c_{30} I_{t}, \\
& I_{t}=a_{t} Y_{t-1},
\end{aligned}
$$

где $I_{t}$ - инвестиции в основные фонды, млрд руб., $a_{t}$ - доля инвестиций в ОФ экономики в ВВП;

9. основные фонды (ОФ) промышленности

$$
\begin{aligned}
& F i_{t}=c_{31} F i_{t-1}+c_{32} I i_{t}, \\
& I i_{t}=a i_{t} I_{t},
\end{aligned}
$$

где $I i_{t}$ - инвестиции в ОФ промышленности, млрд руб., $a i_{t}$ - доля инвестиций в ОФ промышленности в инвестициях в ОФ экономики;

10. основные фонды сектора услуг

$$
\begin{aligned}
& F s_{t}=c_{33} F s_{t-1}+c_{34} I s_{t}, \\
& I s_{t}=a s_{t} I_{t},
\end{aligned}
$$

где $I s_{t}$ - инвестиции в ОФ сектора услуг, млрд руб., $a s_{t}$ - доля инвестиций в ОФ экономики в инвестициях в ОФ экономики;

11. потребление

$$
C_{t}=c_{35}+c_{36} Y_{t} \text {, }
$$

12. отношение средней заработной платы в промышленности к средней заработной плате в финансовой деятельности

$$
w_{t}=c_{37}+c_{38}\left(Y i_{t} / L i_{t}\right) \text {. }
$$

Оценки параметров уравнений системы были получены с использованием данных $[4,8,9]$ (см. табл. 3 в приложении). Результаты моделирования выпуска и численности занятых ИТС свидетельствуют о достаточно высокой точности разработанной модели. Среднеквадратическая ошибка (RMSE) для рассматриваемого периода 2000-2018 гг. составила 
для численности выпуска ИТС $\left(G E_{t}\right)$ приблизительно $2.8 \%$, для численности занятых ИТС $\left(E T_{t}\right)$ - около $2.9 \%$.

Апробация модели для проведения сценарного анализа. Для проведения сценарного анализа возможной несбалансированности спроса и предложения ИТС были рассмотрены 3 сценария (консервативный, базовый, целевой), приведенные в прогнозе социальноэкономического развития Российской Федерации на период до 2024 г. Минэкономразвития [10] (далее - прогноз МЭР). Оценки дополнительного спроса на ИТС (DETt) и предложения труда ИТС (SGEt) были получены с использованием разработанной макромодели.

Основными источниками информации для проведения прогнозов были данные вышеупомянутого прогноза МЭР [10] и данные прогноза Института социологии РАН (далее - ИС РАН) до 2030 г. [11].

Экзогенными переменными являлись темп прироста ВВП, темп прироста промышленных производств, численность занятых. Основные характеристики сценариев 1-3 представлены в табл. 1.

Таблица 1. Изменения показателей согласно базовому, консервативному и целевому сценариям, прогноз социально-экономического развития МЭР [10].

\begin{tabular}{|l|l|c|c|}
\hline Показатель & \multicolumn{1}{|c|}{ Сценарий } & 2020 & 2024 \\
\hline \multirow{2}{*}{$\begin{array}{l}\text { ВВП, темп прироста, к предыдуще- } \\
\text { му году, \% }\end{array}$} & 1 (консервативный) & 1.1 & 2.5 \\
\cline { 2 - 4 } & 2 (базовый) & 1.7 & 3.3 \\
\cline { 2 - 4 } & 3 (целевой) & 2.0 & 3.3 \\
\hline \multirow{2}{*}{$\begin{array}{l}\text { Промышленные производства, темп } \\
\text { прироста, к предыдущему году, \% }\end{array}$} & 1 (консервативный) & 1.7 & 2.2 \\
\cline { 2 - 4 } & 2 (базовый) & 2.4 & 3.1 \\
\cline { 2 - 4 } & 3 (целевой) & 2.8 & 3.3 \\
\hline \multirow{2}{*}{ Численность занятых, млн человек } & 1 (консервативный) & 72.1 & 73.1 \\
\cline { 2 - 4 } & 2 (базовый) & 72.1 & 73.9 \\
\cline { 2 - 4 } & 3 (целевой) & 72.1 & 73.9 \\
\hline
\end{tabular}

При расчете численности выпуска специалистов ИТС для всех рассмотренных сценариев данные по численности выпуска специалистов в целом были взяты из прогноза ИС РАН до 2030 г. [9]. При определении прогнозных оценок предложения труда ИТС, выраженного численностью выпускников инженерно-технических специальностей, учитывается возможный отток специалистов в другие профессии и отрасли после окончания ссузов и вузов. По данным Росстата [12], за период 2013-2017 гг. основная работа была связана с полученной профес- 
сией у 69-73\% выпускников инженерно-технических специальностей, получивших высшее профессиональное образование, и 50-53\% выпускников инженерно-технических специальностей. В работе было сделано предположение, что доля работающих не по полученной профессии ИТС ( $\xi$ ) в среднем составляет $40 \%$.

Результаты проведенного анализа (см. табл. 2) показывают, что для консервативного сценария, характеризующегося относительно более низкими темпами промышленного развития, возможно сохранение некоторого превышения предложения труда ИТС (выпуска специалистов) над спросом, результатом чего может стать увеличение номинальной избыточности предложения труда, и, как следствие, рост доли молодых инженеров и техников, не работающих по специальности после окончания профессионального образования. В то же время для базового и целевого сценариев, незначительно отличающихся друг от друга, при темпах прироста ВВП и промышленного производства около 3\% соответственно в краткосрочном периоде возможно достижение ситуации сбалансированности спроса и предложения все еще при сохранении высокого уровня оттока кадров.

Таблица 2. Результаты оценки степени сбалансированности спроса и предложения труда ИТС $\left(\mathrm{SGE}_{\mathrm{t}} / \mathrm{DET}_{\mathrm{t}}\right)$ для сценариев $1-3$, полученные с использованием модели (1)-(14).

\begin{tabular}{|c|c|c|c|}
\hline Год/ № сценария & Сценарий 1 & Сценарий 2 & Сценарий 3 \\
\hline 2020 & 1.10 & 1.05 & 1.02 \\
\hline 2021 & 1.04 & 0.96 & 0.96 \\
\hline 2022 & 1.14 & 1.02 & 1.01 \\
\hline 2023 & 1.13 & 1.01 & 1.00 \\
\hline 2024 & 1.19 & 1.02 & 1.00 \\
\hline
\end{tabular}

Выводы. Предлагаемый подход, основанный на использовании разработанной макроэкономической модели, дает возможность получить приближенные оценки показателей спроса и предложения ИТС и, соответственно, возможной степени несбалансированности. Для получения более точных оценок необходимо дополнительно учитывать в модели такие показатели, как профессиональная мобильность, миграция и другие социально-экономические факторы, воздействующие на динамику рынка труда ИТС. 


\section{СПИСОК ЛИТЕРАТУРЫ}

1. Larson R.C., Xue Yi. STEM crisis or STEM surplus? Yes and yes // Monthly Labor Review. Bureau of Labor Statistics. May 2015. URL:

http://www.bls.gov/opub/mlr/2015/article/stem-crisis-or-stem-surplus-yes-and-yes.htm

2. Гимпельсон В., Капелюшников Р., Лукьянова А. Спрос на труд и квалификацию в промышленности: между дефицитом и избытком: Препринт WP3/2007/03. - М.: ГУ ВШЭ, 2007.

3. Гуртов В.А., Питухин Е.А., Серова Л.М. Моделирование потребностей экономики в кадрах с профессиональным образованием // Проблемы прогнозирования. 2007. №6. С. 91-107.

4. «О численности и потребности организаций в работниках по профессиональным группам» // Росстат. Выпуски за 2008, 2012, 2014, 2016, 2018 гг.

5. Егоршин А.П., Аблязова Н.О., Гуськова И.В. Состояние и прогнозы развития высшего экономического образования России до 2015 г. // Bonpocbl образования. 2007. № 2. С. 43-55.

6. Гуртов В.А., Серова Л.М., Степусь И.С. Приоритеты экономики: прогнозирование потребностей в кадрах с высшим профессиональным образованием // Университетское управление: практика и анализ, 2011. №4. С.43-51.

7. Гимпельсон, B.E. Нужны ли нашей промышленности квалифицированные работники? История последнего десятилетия : Препринт WP3/2010/04 [Текст] / В. Е. Гимпельсон ; Гос. ун-т - Высшая школа экономики. - М.: Изд. дом Гос. ун-та - Высшей школы экономики, 2010.

8. Российский статистический ежегодник. Выпуски за 2008-2019 гг.

9. База данных World Development Indicators / World Bank. URL: http://datatopics.worldbank.org/world-development-indicators/

10. Прогноз социально-экономического развития Российской Федерации на период до 2024 года. URL: http://economy.gov.ru/minec/activity/sections/macro/prognoz/2019093005

11. Шереги Ф.Э., Арефьев А.Л., Ключарев Г.А., Тюрина И.О. Численность обучающихся, педагогического и профессорско-преподавательского персонала, число образовательных организаций Российской Федерации. (Прогноз до 2020 года и оценка тенденций до 2030 года). М.: Институт социологии РАН, Центр социального прогнозирования и маркетинга, 2015.

12. Итоги выборочного обследования рабочей силы. Росстат. Выпуски за 2013-2017 гг. URL: https://rosstat.gov.ru/compendium/document/13265 
Кочеткова Е.В. Подход к анализу степени сбалансированности.., стр. 162-170

Kochetkova E.V. An approach for engineering labour market..., pp. 162-170

\section{ПРИЛОЖЕНИЕ}

Таблица 3. Оценки параметров макроэкономической модели для анализа возможной степени сбалансированности спроса и предложения труда ИТС (3)-(17).

\begin{tabular}{|c|c|c|c|c|c|c|}
\hline $\begin{array}{l}\text { № урав- } \\
\text { нения }\end{array}$ & $\begin{array}{l}\text { Зависимая } \\
\text { перемен- } \\
\text { ная }\end{array}$ & $\begin{array}{l}\text { Коэф- } \\
\text { фици- } \\
\text { ент }\end{array}$ & Фактор & $\begin{array}{l}\text { Оценка } \\
\text { парамет- } \\
\text { ра }\end{array}$ & $\begin{array}{l}\text { t- } \\
\text { стати- } \\
\text { стика }\end{array}$ & $\mathrm{R}^{2}$ \\
\hline \multirow[t]{7}{*}{$3 a$} & \multirow[t]{7}{*}{$\Delta g e_{t} / g e_{t-1}$} & $c_{1}$ & const & 42.660 & 1.958 & \multirow[t]{7}{*}{0.76} \\
\hline & & $c_{2}$ & $g e_{t-1}$ & $\begin{array}{l}-1.509 \\
\end{array}$ & -2.684 & \\
\hline & & $c_{3}$ & $\Delta g e_{t-1}$ & 1.010 & 1.116 & \\
\hline & & $c_{4}$ & $g s_{t-1}$ & -0.195 & -0.790 & \\
\hline & & $c_{5}$ & $\Delta Y i_{t-5} / Y i_{t-6}$ & 0.169 & 1.127 & \\
\hline & & $c_{6}$ & $D 1$ & 11.965 & 4.103 & \\
\hline & & $c_{7}$ & D2 & 4.756 & 0.302 & \\
\hline \multirow[t]{4}{*}{36} & \multirow[t]{4}{*}{$\Delta g s_{t} / g s_{t-1}$} & $c_{8}$ & const & 59.357 & 7.277 & \multirow[t]{4}{*}{0.76} \\
\hline & & $c_{9}$ & $g e_{t-1}$ & -1.100 & -5.344 & \\
\hline & & $c_{10}$ & $g s_{t-1}$ & -0.676 & -6.464 & \\
\hline & & $c_{11}$ & $\Delta Y s_{t-6} / Y s_{t-7}$ & 0.081 & 1.003 & \\
\hline \multirow[t]{3}{*}{$4 a$} & \multirow[t]{3}{*}{$e t_{t}$} & $c_{12}$ & $g e_{t-1}$ & 0.088 & 2.06 & \multirow[t]{3}{*}{0.93} \\
\hline & & $c_{13}$ & $w_{t}$ & 0.134 & 5.10 & \\
\hline & & $c_{14}$ & D3 & 0.674 & 2.02 & \\
\hline \multirow[t]{2}{*}{5} & \multirow[t]{2}{*}{$\operatorname{Ln}\left[\mathrm{Yi}_{t} / \mathrm{Li} i_{t}\right]$} & $c_{15}$ & const & 2.476 & 4.794 & \multirow[t]{2}{*}{0.85} \\
\hline & & $c_{16}$ & $L n\left[F i_{t} Z_{t} / L i_{t}\right]$ & 0.734 & 9.735 & \\
\hline \multirow[t]{2}{*}{$6 a$} & \multirow[t]{2}{*}{$\operatorname{Ln}\left[Y_{t} / L_{t}\right]$} & $c_{17}$ & const & 3.763 & 5.657 & \multirow[t]{2}{*}{0.59} \\
\hline & & $c_{18}$ & $\operatorname{Ln}\left[F_{t} / L_{t}\right]$ & 0.368 & 3.905 & \\
\hline \multirow[t]{2}{*}{66} & \multirow[t]{2}{*}{$d \operatorname{LnTFP} P_{t}$} & $c_{19}$ & const & -0.005 & -0.834 & \multirow[t]{2}{*}{0.74} \\
\hline & & $c_{20}$ & $\operatorname{Ln}\left[Y i_{t} / L_{t}\right]$ & 0.677 & 6.593 & \\
\hline \multirow[t]{3}{*}{7} & \multirow[t]{3}{*}{$L n\left[Y s_{t} / L s_{t}\right]$} & $c_{21}$ & const & -6.698 & -10.331 & \multirow[t]{3}{*}{0.96} \\
\hline & & $c_{22}$ & $L n\left[F s_{t} / L s_{t}\right]$ & 0.563 & 2.875 & \\
\hline & & $c_{23}$ & $\operatorname{Ln}\left[C_{t}\right]$ & 0.525 & 5.561 & \\
\hline \multirow[t]{2}{*}{$8 a$} & \multirow[t]{2}{*}{$l i_{t}$} & $c_{24}$ & $l i_{t-1}$ & 0.915 & 32.712 & \multirow[t]{2}{*}{0.99} \\
\hline & & $c_{25}$ & $F i_{t-1} Z_{t-1}$ & 2.616 & 2.343 & \\
\hline \multirow[t]{3}{*}{$9 a$} & \multirow[t]{3}{*}{$l_{s_{t}}$} & $c_{26}$ & $l_{t-1}$ & 0.465 & 3.912 & \multirow[t]{3}{*}{0.93} \\
\hline & & $c_{27}$ & $Y_{s_{t-1}} / Y_{t-1}$ & 0.523 & 4.498 & \\
\hline & & $c_{28}$ & D4 & 2.786 & 3.521 & \\
\hline \multirow[t]{2}{*}{$10 a$} & \multirow[t]{2}{*}{$F_{t}$} & $c_{29}$ & $F_{t-1}$ & 0.973 & 181.482 & 0.96 \\
\hline & & $c_{30}$ & $I_{t}$ & 0.572 & 9.654 & \\
\hline $11 a$ & $F i_{t}$ & $c_{31}$ & $F i_{t-1}$ & 0.906 & 7.056 & 0.91 \\
\hline & & $c_{32}$ & $I i_{t}$ & 0.935 & 1.003 & \\
\hline
\end{tabular}


Таблица 3. Продолжение.

\begin{tabular}{|c|c|c|c|c|c|c|}
\hline $\begin{array}{l}\text { № урав- } \\
\text { нения }\end{array}$ & $\begin{array}{l}\text { Зависи- } \\
\text { мая пе- } \\
\text { ременная }\end{array}$ & $\begin{array}{l}\text { Коэф- } \\
\text { фици- } \\
\text { ент } \\
\end{array}$ & Фактор & $\begin{array}{l}\text { Оценка } \\
\text { парамет- } \\
\text { ра }\end{array}$ & $\begin{array}{l}\text { t- } \\
\text { стати- } \\
\text { стика } \\
\end{array}$ & $\mathrm{R}^{2}$ \\
\hline \multirow[t]{2}{*}{$12 a$} & \multirow[t]{2}{*}{$F s_{t}$} & $c_{33}$ & $F_{S_{t-1}}$ & 0.978 & 26.964 & \multirow[t]{2}{*}{0.92} \\
\hline & & $c_{34}$ & $I s_{t}$ & 0.774 & 1.408 & \\
\hline \multirow[t]{2}{*}{13} & \multirow[t]{2}{*}{$C_{t}$} & $c_{35}$ & const & -232.398 & -0.200 & \multirow[t]{2}{*}{0.97} \\
\hline & & $c_{36}$ & $Y_{t}$ & 0.698 & 21.070 & \\
\hline \multirow[t]{2}{*}{14} & \multirow[t]{2}{*}{$w_{t}$} & $c_{37}$ & const & 24.446 & 10.593 & \multirow[t]{2}{*}{0.70} \\
\hline & & $c_{38}$ & $Y i_{t} / L i_{t}$ & 0.008 & 6.132 & \\
\hline
\end{tabular}

\section{THE MODEL FOR ENGINEERING LABOUR MARKET EQUILIBRIUM ANALISYS}

\section{Kochetkova E.V.}

The paper introduces macroeconomic model for analysis and forecasting of demand and supply for engineering and technical personnel. The opportunity of using that model in order to forecast the perspective demand and supply of engineering workforce concerning several scenarios and to evaluate perspective imbalance for engineering labour market in 2020-2024 was shown. 